\title{
THE CHROMIUM OXIDE AND THE VANADIUM OXIDE BAND SPECTRA
}

\author{
By W. F. C. Ferguson ${ }^{1}$
}

\section{ABSTRACT}

With the aid of much more extended measurements on the band heads due to chromium oxide ( $\mathrm{CrO}$ ) and to vanadium oxide ( $\mathrm{VO}$ ) more complete and precise vibrational analyses were made of these spectra. The new equations, representing band heads are found to be:

CrO: $\nu=16,594.6+746.9\left(v^{\prime}+1 / 2\right)-7.9\left(v^{\prime}+1 / 2\right)^{2}-897.5\left(v^{\prime \prime}+1 / 2\right)+6.55\left(v^{\prime \prime}+1 / 2\right)^{2}$; VO: $\nu=17,498.8+864.9\left(v^{\prime}+1 / 2\right)-5.70\left(v^{\prime}+1 / 2\right)^{2}-1,012.3\left(v^{\prime \prime}+1 / 2\right)+5.27\left(v^{\prime \prime}+1 / 2\right)^{2}$.

Additional unassigned bands of $\mathrm{VO}$ are listed.

\section{CONTENTS}

I. Chromium oxide

II. Vanadium oxide

\section{CHROIMIUM OXIDE}

The band spectrum of chromium oxide, $\mathrm{CrO}$, is emitted by an arc containing chromium, in air. Measurements of several band heads have been made by Eder and Valenta, ${ }^{2}$ and others, ${ }^{3}$ and more recently by Kiess and Meggers. ${ }^{4}$ A vibrational analysis of part of the older data was made by Mecke. ${ }^{5}$ In view of the meager data used in that analysis it seemed well to extend the observations of the band heads as far as possible and make a more precise determination of the vibrational constants of the molecule.

The spectrum was obtained by introducing pure chromium metal into a carbon arc. Photographs were taken with low dispersion (about $60 \mathrm{~A}$ per $\mathrm{mm}$ at $6,600 \mathrm{~A}$ ) on a Hilger E2 spectrograph using one glass $60^{\circ}$ prism. Wratten and Wainwright panchromatic plates and especially dyed Eastman 33 plates were used for the visible and infra-red regions of the spectrum, to $9,000 \mathrm{~A}$.

All the band heads that could be measured with certainty were found to belong to the system originally identified by Mecke, ${ }^{6}$ all bands shaded toward longer wave lengths. The intensity distribution among the bands follows a typical Condon ${ }^{7}$ parabola. The

\footnotetext{
1 Assistant professor of physics, New York University. This work was done as guest at the Bureau of Standards.

2 Eder and Valenta, Atlas Typischer Spektren, Wien, 1911.

3 Kayser, u. Konen, Handb. d. Spect., vol. 7, p. 306, 1924; for VO: H. Kayser, Handb., vol. 6, p. 786, 1912.

4 Kiess and Meggers, B. S. Scic. Paper No.'372, 1920.

6 Mecke, Phys. Zeit., vol. 28, p. 514, 1927.

6 Mecke, Phys. Zeit., vol. 28, p. 514, 1927.

7 Condon, Phys. Rev., vol. 28, p. 1182, 1926.
} 
revised equation which represents the positions of all band heads within observational errors is

$$
\begin{array}{cc}
\nu=16,594.6+746.9 \begin{array}{c}
\left(v^{\prime}+1 / 2\right)-7.9\left(v^{\prime}+1 / 2\right)^{2}-897.5\left(v^{\prime \prime}+1 / 2\right) \\
+ \\
+6.55\left(v^{\prime \prime}+1 / 2\right)^{2} .
\end{array}
\end{array}
$$

The duplicity (or multiplicity) of band heads observed by earlier writers does not appear with the low dispersion used here; so the following measurements are of the shortest wave length heads in all probability of multiple bands. Older data included but nine more

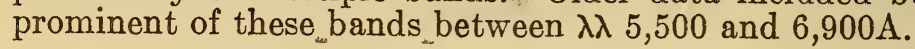

\begin{tabular}{|c|c|c|c|c|}
\hline$v^{\prime}, v^{\prime \prime}$ & Intensity & $\lambda$ (I. A.) & $\begin{array}{l}\nu\left(\mathrm{cm}^{-1}\right) \\
(\text { Obs. })\end{array}$ & $\begin{array}{c}\nu\left(\mathrm{cm}^{-1}\right) \\
\text { (Obs. - Calc.) }\end{array}$ \\
\hline 3,0 & & $5,356.4$ & 18,664 & -1 \\
\hline $\begin{array}{l}2,0 \\
3,1 \\
3\end{array}$ & 3 & $\begin{array}{l}5,564.2 \\
5,623.1\end{array}$ & $\begin{array}{l}17,967 \\
17,779\end{array}$ & $\begin{array}{c}2 \\
-2\end{array}$ \\
\hline $\begin{array}{l}1,0 \\
2,1 \\
3,2\end{array}$ & $\begin{array}{l}4 \\
1 \mathrm{~L} \\
2\end{array}$ & $\begin{array}{l}5,795.5 \\
5,852.8 \\
5,914.5\end{array}$ & $\begin{array}{l}17,250 \\
17,081 \\
16,903\end{array}$ & $\begin{array}{r}0 \\
0 \\
-6\end{array}$ \\
\hline $\begin{array}{l}0,0 \\
2,2 \\
3,3\end{array}$ & $\begin{array}{l}6 \\
2 \\
1\end{array}$ & $\begin{array}{l}6,052.3 \\
6,168.1 \\
6,230.4\end{array}$ & $\begin{array}{l}16,518 \\
16,208 \\
16,046\end{array}$ & $\begin{array}{l}-1 \\
-2 \\
-5\end{array}$ \\
\hline $\begin{array}{l}0,1 \\
1,2\end{array}$ & $\begin{array}{l}3 \\
2\end{array}$ & $\begin{array}{l}6,395.0 \\
6,451.5\end{array}$ & $\begin{array}{l}15,633 \\
15,496\end{array}$ & $\begin{array}{r}-2 \\
2\end{array}$ \\
\hline $\begin{array}{l}0,2 \\
1,3\end{array}$ & $\begin{array}{l}4 \\
3\end{array}$ & $\begin{array}{l}6,771.8 \\
6,829.2\end{array}$ & $\begin{array}{l}14,763 \\
14,639\end{array}$ & $\begin{array}{l}0 \\
3\end{array}$ \\
\hline $\begin{array}{l}0,3 \\
1,4 \\
2,5 \\
3,6 \\
4,7\end{array}$ & $\left(\begin{array}{l}2 \mathrm{~d} \\
2 \\
1 \\
1 \\
0 \\
0\end{array}\right.$ & $\begin{array}{l}7,187.1 \\
7,249.1 \\
7,313.8 \\
7,375.3 \\
7,439.5\end{array}$ & $\begin{array}{l}13,910 \\
13,791 \\
13,669 \\
13,555 \\
13,438\end{array}$ & $\begin{array}{r}5 \\
0 \\
-5 \\
0 \\
5\end{array}$ \\
\hline $\begin{array}{l}1,5 \\
2,6 \\
3,7 \\
4,8\end{array}$ & $\begin{array}{l}0 \\
1 \\
1 \\
1\end{array}$ & $\begin{array}{l}7,713.9 \\
7,778.1 \\
7,842.8 \\
7,908.0\end{array}$ & $\begin{array}{l}12,960 \\
12,853 \\
12,747 \\
12,642\end{array}$ & $\begin{array}{r}1 \\
-2 \\
-2 \\
2\end{array}$ \\
\hline 3,8 & $1 \mathrm{~d}$ & $8,360.3$ & 11,958 & 2 \\
\hline
\end{tabular}

TABLE 1.-Heads of chromium oxide bands

$\mathrm{L}$, line superposed.

d, diffuse.

Calc., calculated values obtained from formula given above.

\section{VANADIUM OXIDE}

The band spectrum of vanadium oxide, VO, is emitted by an arc containing vanadium, in air. The lack of data $8,9,10$ for a more complete analysis ${ }^{11}$ of this spectrum led to further investigation; as in the case of $\mathrm{CrO}$.

The spectrum was obtained by introducing vanadium chloride into a carbon arc. Low dispersion photographs were taken, as in section I, of the visible and infra-red regions. All the bands are shaded toward longer wave lengths.

The more intense of the bands measured extend the system for which the vibrational analysis was initiated by Mecke. ${ }^{12}$

8 Eder and Valenta, Atlas Typischer Spektren, Wien, 1911.

Kayser u. Konen, Hanb. d. Spect., vol. 7. p. 306, 1924; for VO: H. Kayser, Handb., vol. 6, p. 786, 1912.,

10 Kiess and Meggers, B. S. Sci. Paper No. 372, 1920.

11 Mecke, Phys. Zeit., vol. 28, p. 514, 1927.

12 Mecke, Phys. Zeit., vol. 28, p. 514, 1927. 견 
Intensity distribution among the bands is, as might be expected from the ratio of coefficients of $v^{\prime}$ and $v^{\prime \prime}$, a typical distribution. Duplicity (or multiplicity) of band heads, observable under high dispersion, ${ }^{13}$ did not appear on the spectrograms, therefore the present measurements are of the shortest wave length heads in all probability of multiple bands. The new equation which represents the positions of band heads in this system, called system 1, within observational errors, is

$$
\begin{gathered}
\nu=17,498.8+864.9\left(v^{\prime}+1 / 2\right)-5.70\left(v^{\prime}+1 / 2\right)^{2} \\
-1,012.3\left(v^{\prime \prime}+1 / 2\right)+5.27\left(v^{\prime \prime}+1 / 2\right)^{2}
\end{gathered}
$$

Measurements of the band heads are given in Table 2. Older data included but four of these bands: $(1,0),(0,0),(0,1),(0,2)$.

\begin{tabular}{|c|c|c|c|c|}
\hline$v^{\prime}, v^{\prime \prime}$ & Intensity & $\lambda($ I. A.) & $\begin{array}{c}\nu\left(\mathrm{cm}^{-1}\right) \\
(\mathrm{Obs} .)\end{array}$ & $\begin{array}{c}\nu\left(\mathrm{cm}^{-1}\right) \\
\text { (Obs.-Calc.) }\end{array}$ \\
\hline $\begin{array}{l}4,0 \\
5,1 \\
6,2 \\
7,3\end{array}$ & $\begin{array}{l}2 \\
2 \mathrm{I} \\
2 \mathrm{I} \\
2\end{array}$ & $\begin{array}{l}4,812.6 \\
4,858.2 \\
4,904.7 \\
4,951.3\end{array}$ & $\begin{array}{l}20,773 \\
20,578 \\
20,383 \\
20,191\end{array}$ & $\begin{array}{l}2 \\
1 \\
1 \\
4\end{array}$ \\
\hline $\begin{array}{l}3,0 \\
4,1 \\
5,2\end{array}$ & $\begin{array}{l}3 \\
4 \\
3 \mathrm{~L}\end{array}$ & $\begin{array}{l}5,011.1 \\
5,057.3 \\
5,104.3\end{array}$ & $\begin{array}{l}19,950 \\
19,768 \\
19,586\end{array}$ & $\begin{array}{r}-1 \\
-1 \\
0\end{array}$ \\
\hline $\begin{array}{l}2,0 \\
3,1 \\
4,2\end{array}$ & $\begin{array}{l}4 \\
4 \\
2\end{array}$ & $\begin{array}{l}5,228.4 \\
5,275.3 \\
5,323.6\end{array}$ & $\begin{array}{l}19,121 \\
18,951 \\
18,779\end{array}$ & $\begin{array}{l}0 \\
1 \\
1\end{array}$ \\
\hline $\begin{array}{l}1,0 \\
2,1\end{array}$ & $\begin{array}{l}5 \\
2\end{array}$ & $\begin{array}{l}5,469.2 \\
5,516.3\end{array}$ & $\begin{array}{l}18,279 \\
18,123\end{array}$ & $\begin{array}{l}0 \\
4\end{array}$ \\
\hline $\begin{array}{l}0,0 \\
2,2\end{array}$ & ${ }_{3}^{4} \mathrm{~L}$ & $\begin{array}{l}5,737.3 \\
5,837.8\end{array}$ & $\begin{array}{l}17,425 \\
17,125\end{array}$ & $\begin{array}{r}0 \\
-3\end{array}$ \\
\hline 0,1 & $4 \mathrm{~L}$ & $6,087.3$ & 16,423 & 0 \\
\hline $\begin{array}{l}0,2 \\
1,3 \\
2,4\end{array}$ & ${ }_{1}^{2} \mathrm{~L}$ & $\begin{array}{l}6,477.8 \\
6,531.6 \\
6,588.4\end{array}$ & $\begin{array}{l}15,433 \\
15,306 \\
15,174\end{array}$ & $\begin{array}{r}1 \\
1 \\
-3\end{array}$ \\
\hline $\begin{array}{l}0,3 \\
1,4 \\
2,5 \\
3,6 \\
4,7\end{array}$ & $\begin{array}{l}3 \\
3 \\
3 \\
1 \\
0 \mathrm{~d}\end{array}$ & $\begin{array}{l}6,916.6 \\
6,974.5 \\
7,034.4 \\
7,091.3 \\
7,149.1\end{array}$ & $\begin{array}{l}14,454 \\
14,334 \\
14,212 \\
14,098 \\
13,984\end{array}$ & $\begin{array}{r}3 \\
-1 \\
-5 \\
-1 \\
4\end{array}$ \\
\hline $\begin{array}{l}0,4 \\
1,5 \\
2,6 \\
3,7\end{array}$ & $\begin{array}{l}1 \\
3 \\
2 \\
0 \mathrm{~d}\end{array}$ & $\begin{array}{l}7,416.9 \\
7,474.0 \\
7,533.7 \\
7,593.2\end{array}$ & $\begin{array}{l}13,479 \\
13,376 \\
13,270 \\
13,166\end{array}$ & $\begin{array}{r}-2 \\
1 \\
2 \\
5\end{array}$ \\
\hline $\begin{array}{l}(0,5) \\
(1,6)\end{array}$ & $\underset{\mathrm{m}}{\mathrm{m}}$ & & $\begin{array}{l}112,522 \\
112,426\end{array}$ & \\
\hline $\begin{array}{l}0,6 \\
1,7\end{array}$ & $\begin{array}{ll}1 \mathrm{~L} \\
1 \mathrm{~d}\end{array}$ & $\begin{array}{l}8,639.9 \\
8,701.6\end{array}$ & $\begin{array}{l}11,571 \\
11,489\end{array}$ & $\begin{array}{r}-2 \\
1\end{array}$ \\
\hline
\end{tabular}

TABLE 2.-Vanadium oxide band heads of system 1

L, line superposed.

$\mathrm{d}$, diffuse.

m, masked by strong band, 12,534 .

Calc., calculated from formula, above.

1 Calc. only.

In addition to system 1 there are 45 bands, most probably due to VO, which could not be assigned to any system. Fourteen of these

13 Eder and Valenta, Atlas Typischer Spektren, Wien, 1911.

$99675-32-5$ 
bands form four sequences which appear from their first differences to be related to system 1. However, no relation was found. Nor do these sequences comprise a satisfactory new system. In it there would be no band in a negative sequence if, as seems possible, the 0 , $+1,+2,+3$ sequences be represented in the following order:

$\begin{array}{llll}14,503 & 13,559 \mathrm{~L} & 12,629 \mathrm{~L} & 11,708 \mathrm{~L} \\ 14,381 & 13,448 & 12,534 & 11,625 \\ 14,261 & 13,340 & & \\ 14,139 & & & \\ 14,023 \mathrm{~L} & 13,121 & & \\ 13,899 & & & \\ \text { L, line superposed. } & \end{array}$

Additional band heads that were measured, generally of less intensity, are listed below:

$\begin{array}{lll}20,000 & 13,629 & 12,651 \mathrm{~L} \\ 17,288 \mathrm{~L} & 13,594 \mathrm{~L} & 12,089 \\ 17,248 \mathrm{~L} & 13,521 & 12,036 \\ 16,831 & 13,500 & 12,018 \\ 15,671 & 13,431 & 11,934 \\ 15,547 & 13,409 & 11,661 \mathrm{~L} \\ 14,677 \mathrm{~L} & 13,301 & 11,595 \mathrm{~L} \\ 14,556 & 12,820 \mathrm{~L} & 11,550 \mathrm{~L} \\ 14,411 & 12,710 & 11,414 \\ 14,291 & 12,661 \mathrm{~L} & \\ 14,082 & 12,591 \mathrm{~L} & \\ \text { L, confused with line. } & \end{array}$

The writer wishes to thank the members of the spectroscopy section, especially Dr. W. F. Meggers, for kindnesses extended to him while at the Bureau of Standards.

Washington, December 22, 1931. 